\title{
Acceleration and heating processes in a collapsing magnetic trap
}

\author{
M. Karlický and T. Kosugi
}

\author{
Astronomical Institute of the Academy of Sciences of the Czech Republic, 25165 Ondřejov, Czech Republic \\ Institute of Space and Astronautical Sciences, 3-1-1 Yoshinodai, Sagamihara, Kanagawa 229-8510, Japan \\ Received 16 September 2003 / Accepted 23 February 2004
}

\begin{abstract}
We study the acceleration processes in a collapsing magnetic trap formed in the cusp structure of the flare model, using a test-particle numerical method. Coulomb collisions and scattering are included. It was found that if the trap collapse is sufficiently fast and the energies of the injected electrons are sufficiently high, thus overcoming the collisional losses, electrons can be accelerated in this secondary acceleration process to very high energies depending on the initial magnetic trap ratio $R=B_{\max } / B_{\text {init }}$. The computations, made for $R=10$ and $R=100$ with isotropically injected 5-28.4 keV electrons and a background plasma density of about $n_{\mathrm{e}}=10^{10} \mathrm{~cm}^{-3}$, show that the high-energy electrons are accumulated in the central part of the collapsing magnetic trap where their velocities are nearly perpendicular to the magnetic field. This effect gives us a new possibility to explain the formation of loop-top sources observed in hard X-ray and radio emission. A further interesting aspect is that these electrons later on escape from this collapsing trap because its trap ratio decreases to $R \rightarrow 1$. The time evolution of the energy of the trapped electrons and their energy flux at the end points of the trap (footpoints) are computed for cases without and with collisions. The effect of collisions on the energy spectrum of the accelerated electrons is also shown. The $\mathrm{X}$-ray spectra along the collapsing trap are evaluated. Finally, we suggest a test of this model considering radio waves in the decimetric frequency range.
\end{abstract}

Key words. plasmas - Sun: flares - Sun: particle emission - acceleration of particles

\section{Introduction}

An extended review of particle acceleration processes in solar flares was recently published by Aschwanden (2002). It presents a magnetic field topology of acceleration regions ( 2 and 3 dimensional, bipolar, tripolar and quadrupolar) as well as basic acceleration mechanisms (direct current (DC) electric field, stochastic, and shock wave accelerations) (see also references therein).

In the standard 2-D solar flare model (e.g. Priest \& Forbes $2000)$ it is assumed that vertical, oppositely oriented magnetic fields form the current sheet in which the magnetic field reconnection takes place and particles are accelerated in the DC electric field (Litvinenko 1996). This acceleration process is considered to be the primary one and for further particle acceleration various secondary acceleration processes have been proposed: a) acceleration in the MHD turbulence generated in fast plasma reconnection outflows (LaRosa \& Moore 1993; Miller et al. 1996); b) shock-drift (Holman \& Pesses 1983); c) diffusive shock acceleration (Cargill et al. 1988); d) firstorder Fermi acceleration in a fast termination shock (Tsuneta \& Naito 1998); and e) first-order Fermi acceleration in a mirrortrap (Somov \& Kosugi 1997).

Send offprint requests to: M. Karlický, e-mail: karlicky@asu.cas.cz
Among secondary acceleration processes there is one acceleration process that is not commonly known, but that naturally follows from the motion of the magnetic field lines in the cusp-structure below the reconnection current sheet and above the underlying flare loops. Namely, in the reference system of moving magnetic field lines and flowing plasma the magnetic field changes in time. This system of moving magnetic field lines at this position was called by Somov \& Kosugi (1997) and Jakimiec (2002) the collapsing magnetic trap and it was proposed as an efficient accelerator of particles. While Somov \& Kosugi (1997) simplified the process by a model with two moving magnetic mirrors located at the termination shock, Jakimiec (2002) emphasized the betatron type of particle acceleration in this configuration.

In the betatron mechanism particles are accelerated in the electric field $\boldsymbol{E}$ generated by time variations of the magnetic field $\boldsymbol{B}$ as follows from Faraday's law $\nabla \times \boldsymbol{E}=-\partial \boldsymbol{B} / \partial t$ (Swann 1933; Berger et al. 1958). This type of acceleration was considered, for example, by Brown \& Hoyng (1975) and by Emslie (1981) in connection with non-thermal and thermal hard X-ray sources. Futhermore, the betatron process was invoked also for the deceleration of electrons in expanding radio sources (Bruggmann et al. 1994).

In the present paper, we want to show the importance of this acceleration process, especially because it can be connected 


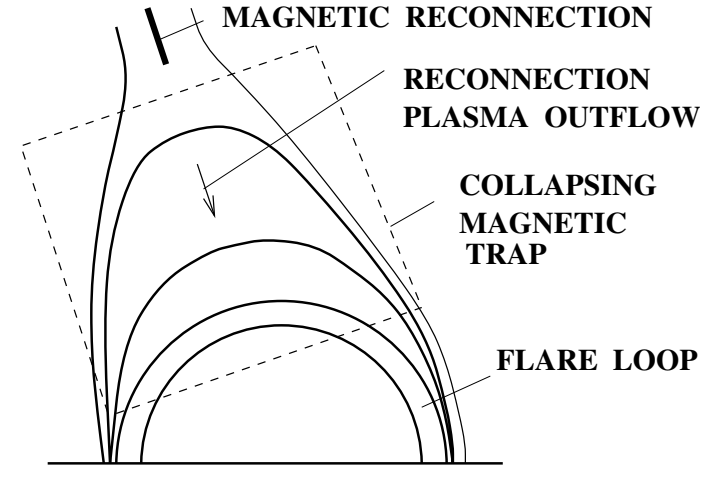

Fig. 1. The scheme of the collapsing magnetic trap in the standard 2-D flare model.

with or even can explain (see the following) phenomena such as the loop-top hard X-ray source (Masuda et al. 1996), loop-top super hot plasma (Tsuneta et al. 1997), and nonthermal collimated ejection (Nakajima \& Yokoyama 2002).

First, the model relation, expressing the evolution of a magnetic field line in the cusp-structure, is presented. Using a test particle approach, the betatron acceleration, collisional losses and scattering of electrons in the background plasma are included in the model. Then the results of numerical simulations are shown. The betatron (adiabatic) heating is mentioned. The diagnostics of these processes on radio waves in the decimetric frequency range is proposed. Finally, the basic physical aspects of these processes are summarized and discussed.

\section{Model}

As shown in the scheme of the standard 2-D flare model (Fig. 1) (Priest \& Forbes 2000), the reconnected magnetic field lines and plasma particles move together from the reconnection space ( $X$-point) towards the flare loop. Plasma particles gyrate around these moving magnetic field lines, under the influence of an increasing magnetic field (especially in the centre of the collapsing magnetic trap) and thus they are accelerated. Generally, the properties (length and magnetic field values) of these magnetic field lines and their evolutions could be obtained from 2-D or 3-D MHD numerical simulations. But this is not a simple task. Therefore for an estimation of the efficiency of this acceleration process we express the time dependence of the magnetic field strength along the magnetic field line in a reference system moving with this line by the following schematic way.

In the initial state of evolution of the magnetic field line, the magnetic field strength along the line has to be similar to that near the $X$-point magnetic field configuration (Priest 1982), where the magnetic field vector can be expressed as

$\boldsymbol{B}=Y \boldsymbol{x}+\alpha^{2} X \boldsymbol{y}$,

where $\alpha$ is a parameter, $X$ and $Y$ are spatial coordinates in the reference system where the zero point ( $X=0, Y=0)$ corresponds to the $X$-point. The magnetic field along a specific magnetic field line $\left(Y^{2}-\alpha^{2} X^{2}=C\right)$ can thus be written as:

$$
|\boldsymbol{B}|=\sqrt{C+\alpha^{2} X^{2}+\alpha^{4} X^{2}},
$$

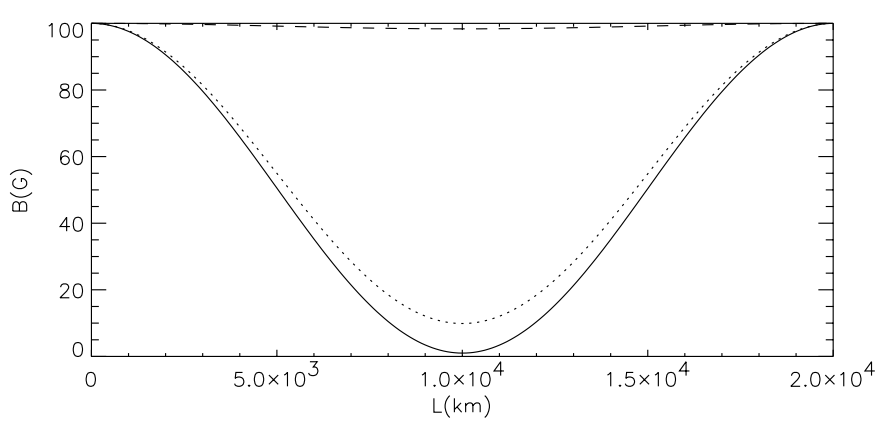

Fig. 2. The magnetic field profiles in the reference system of moving magnetic field lines in the collapsing magnetic trap at $t_{0}^{100}=0.01 \mathrm{~s}$ (the initial state for the trap ratio $R=100$ - the full line), at $t_{0}^{10}=0.1033 \mathrm{~s}$ (the initial state for the trap ratio $R=10$ - the dotted line), and at $t_{0}^{10}+4 \mathrm{~s}$ (the dashed line).

where $C$ is the constant specifying the magnetic field line. Analyzing this relation, it can be seen that the magnetic field strength at specific magnetic field line has a minimum at the point $X=0$ and it increases monotonically for both positive and negative values of $X$. Moreover, the minimum increases with an increase of the parameter $C$, i.e. with an increase of a distance of the magnetic field line from the $X$-point.

On the other hand, in the final state of the collapsing trap the magnetic field should be similar to the flare loop with the potential magnetic field. A shrinkage of the magnetic field line just after its reconnection in the $X$-point is faster than that in the stage close to final one because the flare loop represents an obstacle for a reconnection plasma outflow. Furthermore, magnetic field lines are anchored in the photosphere, where the magnetic field is much higher than that close to the $X$-point.

To fulfil approximately all these demands, in the following computations the time evolution of the magnetic field along one magnetic field line in the reference system of moving plasma is expressed in the following form:

$B(t)=B_{0}\left\{1-\frac{1}{2}\left[1-\cos \left(\frac{2 \pi x}{L}\right)\right] \mathrm{e}^{-\frac{t_{0}+t}{t_{c}}}\right\}$

where $t$ is the time, $t_{0}$ is the "initial" time (see the following), $B_{0}$ is the magnetic field at the footpoints of the magnetic field line, $x$ is the space coordinate, $L$ is the loop length, $L / 2$ is the position of the centre of the magnetic field line (i.e. also the position of the $X$-point with $x=X+L / 2)$ and $t_{\mathrm{c}}$ is the characteristic time of the collapse. From this relation it follows that at the positions $x=0$ and $x=L$ (footpoints of the magnetic field line) the magnetic field strength is kept constant $\left(B=B_{0}\right)$. On the other hand, at other positions between $x \equiv(0-L)$ the magnetic field strength increases with time, and for $t / t_{\mathrm{c}} \gg 1$ the magnetic field strength is close to $B_{0}$; but still with a minimum of the magnetic field at the line centre - loop top (the magnetic field profile approximately simulating the final potential magnetic field). The maximum changes are in the centre of the collapsing trap (see Fig. 2). The exponentional function in the relation (3) expresses a shrinkage of the magnetic field line. The curvature of the magnetic field line is neglected in the following computations. The model relation (3) is a mathematical approximation which can be generally modified on the base of a comparison of model results with observations. 
At a specific time the spatial magnetic field profile has the form of a magnetic bottle (trap) with magnetic mirrors at the positions $x=0$ and $x=L$. For such a trap we can define the trap (mirror) ratio as that of the maximum magnetic field at the footpoints of the magnetic trap to that in the centre of the trap $\left(R=B_{0} / B(x=L / 2)\right)$. According to Eq. (3) this ratio decreases with time, and at $t / t_{\mathrm{c}} \gg 1$ the ratio is $R \rightarrow 1$.

In the present case, the parameters are chosen as $B_{0}=$ $100 \mathrm{G}, L=2 \times 10^{4} \mathrm{~km}, t_{\mathrm{c}}=1 \mathrm{~s}$. Generally, the length of the magnetic field line $L$ is a function of time. But by selecting a fixed length $L$ we neglect in the following the effect of shortening of the magnetic field line during its motion in the cusp structure. The initial trap ratio can be chosen by a specification of the "initial" time. Thus, for an initial trap ratio $R=B_{\max } / B_{\text {init }}(x / L=0.5)=10$ and $R=100$ we define the initial time as $t_{0}^{10}=0.1033 \mathrm{~s}$ and $t_{0}^{100}=0.01 \mathrm{~s}$, respectively. According to relation (3) these times define the initial magnetic field profiles (see Fig. 2).

In the following only the acceleration of electrons will be considered. The electrons are described as a cloud of numerical particles having prescribed momentum $p$ (energy $E$ ) and a cosine of the pitch angle $\mu$ expressing the angle between the direction of motion of the electron and the magnetic field. Furthermore, each electron can have its own "weight". Namely, one numerical electron can represent a different number of real electrons. This reduces the demand for a large amount of numerical electrons. Clearly, in an interpretation of the computational results it is necessary to take this "weight" into account.

We used the same approach as in our previous papers (Karlický 1993; Karlický \& Henoux 1993), but now for high energies the relativistic corrections are included. The trajectories of all numerical electrons along magnetic field lines are computed as follows:

$x_{\text {new }}=x_{\text {old }}+v_{\|} \Delta t$,

where $v_{\|}$is the electron velocity along the magnetic field, and $x_{\text {new }}$ and $x_{\text {old }}$ are the new and old position of the electron during the time step $\Delta t$. For both the magnetic mirroring and betatron effects the conservation of the electron magnetic moment

$$
\frac{p^{2}\left(1-\mu^{2}\right)}{B}=\text { const. }
$$

is used. First a change of the pitch angle due to the mirroring effect is computed and then at the specific electron position the betatron effect is calculated, i.e. the change of the perpendicular component of the momentum $p_{\perp}$ due to a time change of the magnetic field.

The energy losses and pitch angle scattering of electrons due to Coulomb collisions with the surrounding plasma are calculated by the Monte Carlo method as in the paper of Bai (1982), i.e. the following formulae are used:

$$
\begin{aligned}
\Delta E & =\frac{4 \pi n_{\mathrm{e}} e^{4} \Lambda L_{\mathrm{p}}}{m_{\mathrm{e}} v^{2}}, \\
\left\langle\theta^{2}\right\rangle & =\frac{16 \pi n_{\mathrm{e}} e^{4} \Lambda L_{\mathrm{p}}}{\gamma^{2} m_{\mathrm{e}}^{2} v^{4}},
\end{aligned}
$$

where $E$ is the electron energy, $\left\langle\theta^{2}\right\rangle$ is the deflection angle, $L_{\mathrm{p}}$ is the distance which an electron travels in a plasma, $m_{\mathrm{e}}$ is the electron mass, $\Lambda$ is the Coulomb logarithm, $n_{\mathrm{e}}$ is the density of the surrounding plasma, $\gamma$ is the Lorentz factor, and $v$ is the electron velocity.

As concerns the plasma density in the collapsing magnetic trap, an increase of the magnetic field means compression and thus increase of the plasma density. In a highly ionized plasma magnetic field lines are "frozen" in the plasma, which here leads to the relation

$\frac{B(t)}{B_{0}}=\frac{n(t)}{n_{0}}$

where $n_{0}$ is the plasma density in the flare loop footpoints (Fig. 1). To satisfy this relation in a simple way, the plasma density along a moving magnetic field line in the cusp-structure above the flare loop is assumed to be expressed in a similar way as the magnetic field, i.e.

$n(t)=n_{0}\left\{1-\frac{1}{2}\left[1-\cos \left(\frac{2 \pi x}{L}\right)\right] \mathrm{e}^{-\frac{t_{0}+t}{t_{\mathrm{c}}}}\right\}$

\section{Results of simulations}

First, the computations were made without Coulomb collisions, for an initial trap ratio $R=10$. In the initial state 1000 numerical electrons isotropically distributed (the number of electrons is constant in $\mu$ ) and monoenergetic in energy (10 keV) were injected in the centre of the collapsing magnetic trap $(x=L / 2)$ into the initial magnetic field (relation (3) for $t_{0}^{10}$ ). It is assumed that these electrons were pre-accelerated by a primary acceleration process in the current sheet. To have full 3-D isotropy of the electrons and to save computing time, the numerical electrons have different "weights". Using the cylindrical symmetry of the problem the electron with a specific value of $\mu$ has the "weight" $2 \pi \sqrt{1-\mu^{2}}$. This weighting of electrons is taken into account in the following computations of distribution functions.

Then the acceleration in the collapsing magnetic trap (along a moving magnetic field line in the cusp-structure) was computed. Results of these computations at two times $\left(t_{0}^{10}+1 \mathrm{~s}\right.$ and $t_{0}^{10}+4 \mathrm{~s}$ ) are shown in Figs. 3 and 4 . The time $t_{0}^{10}+4 \mathrm{~s}$ represents nearly the end of the collapse, when a specific magnetic field line touches the flare loop. As seen in Fig. 3, which shows the distribution of numerical electrons in energy-position space (each point in the figure corresponds to one numerical electron), the electrons are bouncing between the magnetic mirrors of the collapsing trap. As expected the electrons with $\mu$ (the cosine of the pitch angle) $\approx 0$ are the most effectively accelerated, thus forming a cloud of energetic (up to $100 \mathrm{keV}$ ) electrons in the centre of the collapsing trap. Their energy is much greater perpendicular to the magnetic field than parallel. On the other hand, the electrons with lower pitch angles are accelerated with lower efficiency because they spent some time at positions with smaller variations of the magnetic field. The electrons moving nearly along magnetic field lines even escape immediately from the trap. The above mentioned accumulation effect in space is also associated with the accumulation effect in the $\mu$ space for $\mu$ 

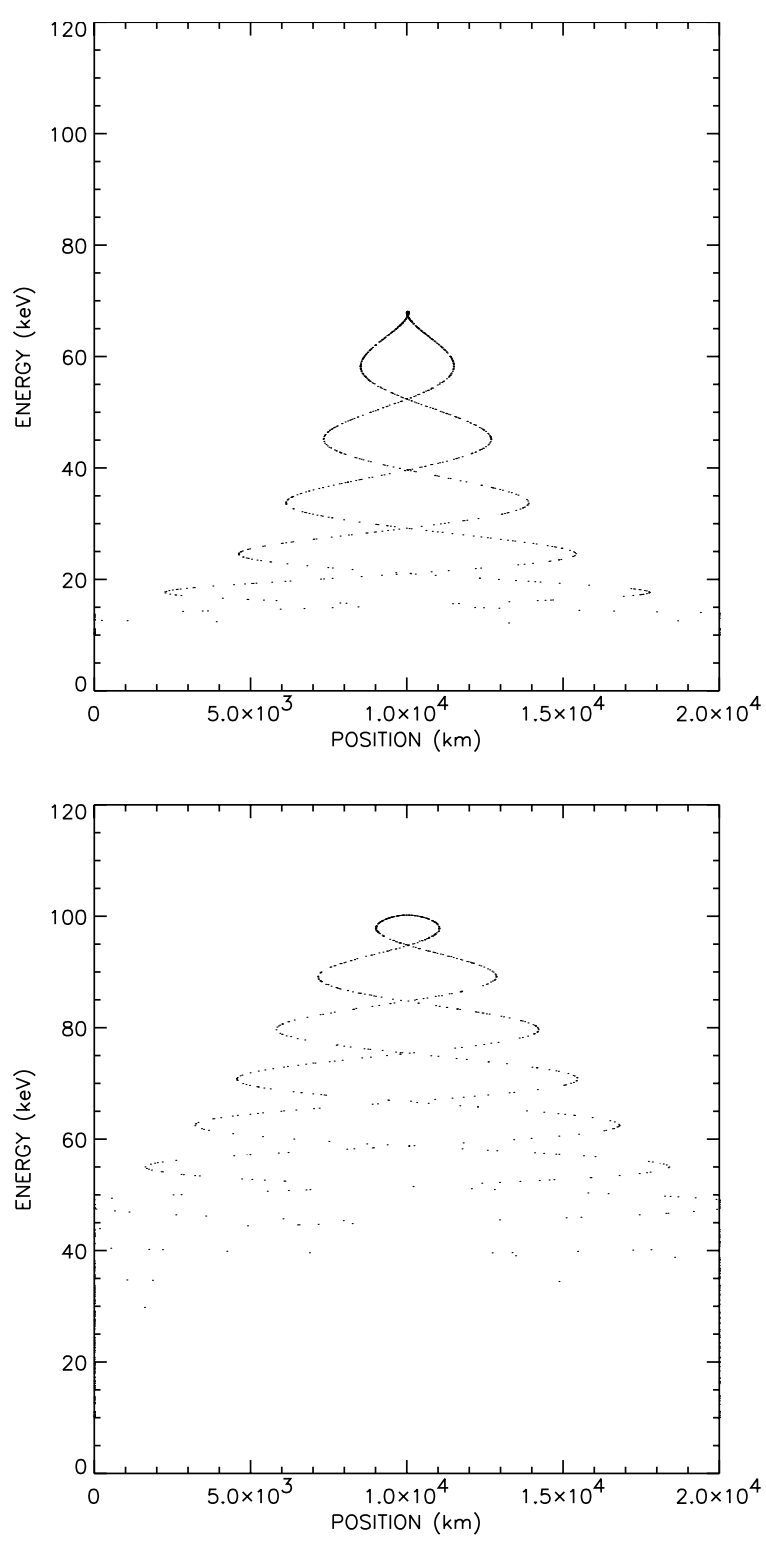

Fig. 3. The distribution of numerical electrons in energy-position space. Each point in the figure corresponds to an individual numerical electron. Positions are expressed along the magnetic field line in the collapsing trap. Upper panel: the state at $t_{0}^{10}+1 \mathrm{~s}$. Lower panel: the state at $t_{0}^{10}+4 \mathrm{~s}$. The initial energy of the electrons is $10 \mathrm{keV}$. The initial trap ratio is $R=10$.

close to zero (see Fig. 4). Namely, the increase of the pitch angle changes the mirror reflection points.

Similar computations were made, but for the higher initial trap ratio $R=100$, i.e. for $t_{0}^{100}=0.01 \mathrm{~s}$, and for more energetic electrons $(28.4 \mathrm{keV}$ corresponding to the velocity $=c / 3$, where $c$ is the speed of light). The electrons were one again injected isotropically at $x=L / 2$. The results are similar to the previous results, but the energies are in the relativistic range (up to $1.4 \mathrm{MeV}$ ), see Figs. 5 and 6.

To describe the acceleration processes in more detail we increased the number of numerical electrons to 10000 and we made computations of the time evolution of the energy of trapped electrons, the number of electrons in the trap, and the
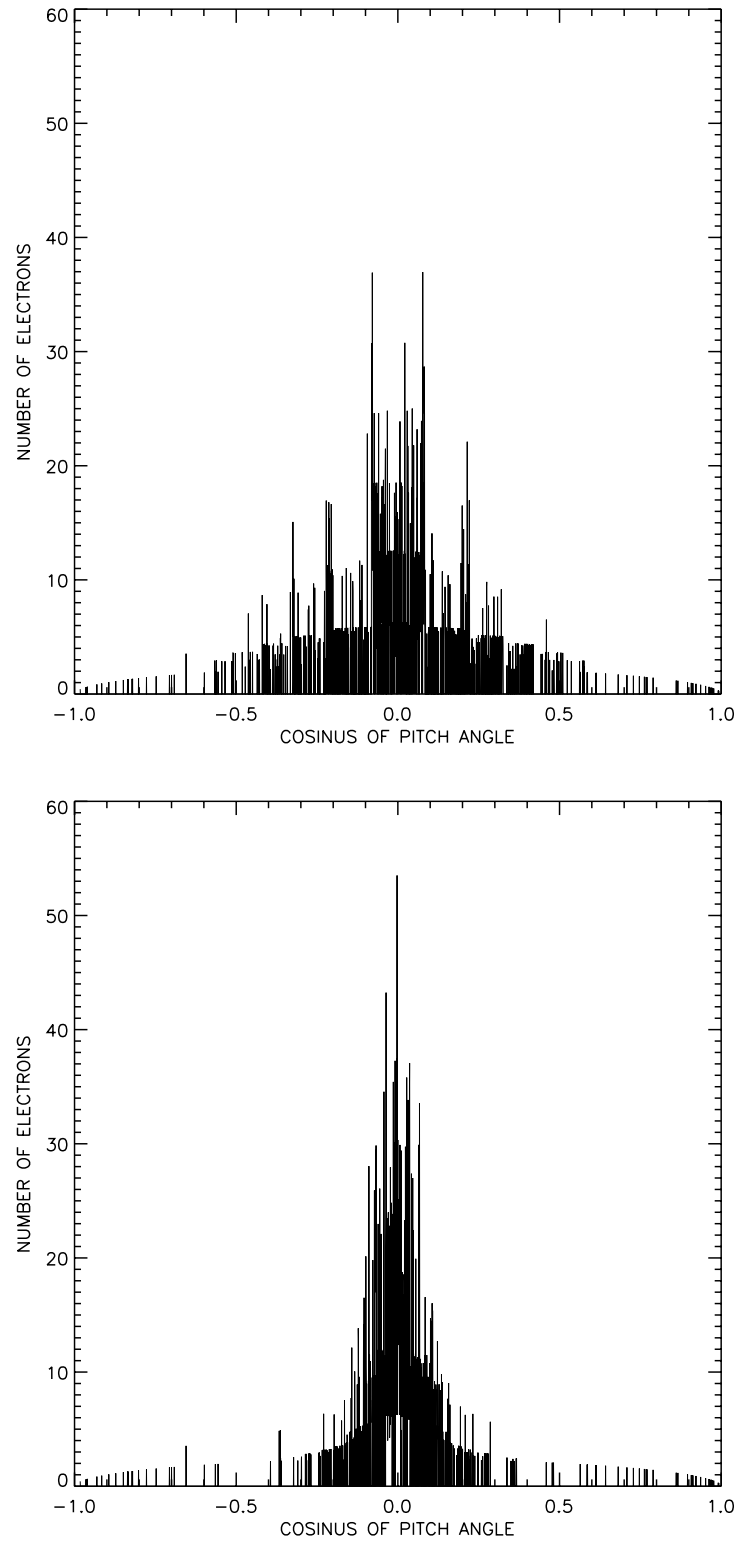

Fig. 4. The distribution of the cosine of the pitch angle of electrons $(\mu)$ in the collapsing magnetic trap at $t_{0}^{10}+1 \mathrm{~s}$ (upper panel), and at $t_{0}^{10}+4 \mathrm{~s}$ (lower panel). The initial energy is $10 \mathrm{keV}$. The initial trap ratio is $R=10$. The electron number includes the weight of the numerical electrons.

energy and integrated energy fluxes at the end point (footpoint) of the collapsing trap. All values were normalized to the initial electron number $N_{0}$. This type of normalization means, e.g. for the energy of trapped electrons, that if no electrons escape from the trap then this normalized energy equals the average electron energy. This type of normalization describes a global evolution of the energy content in the trap, i.e. it takes into account the effect of the decrease of the number of electrons in the trap. The results of the computations for the case without collision (full lines) and with collisions (dashed lines) are presented in Fig. 7. The fluctuations seen in the time profiles, especially for the energy flux, are due to the limited number of electrons used in the computations. In the panel for the number of electrons in the trap only the electrons with non-zero energy are shown. 

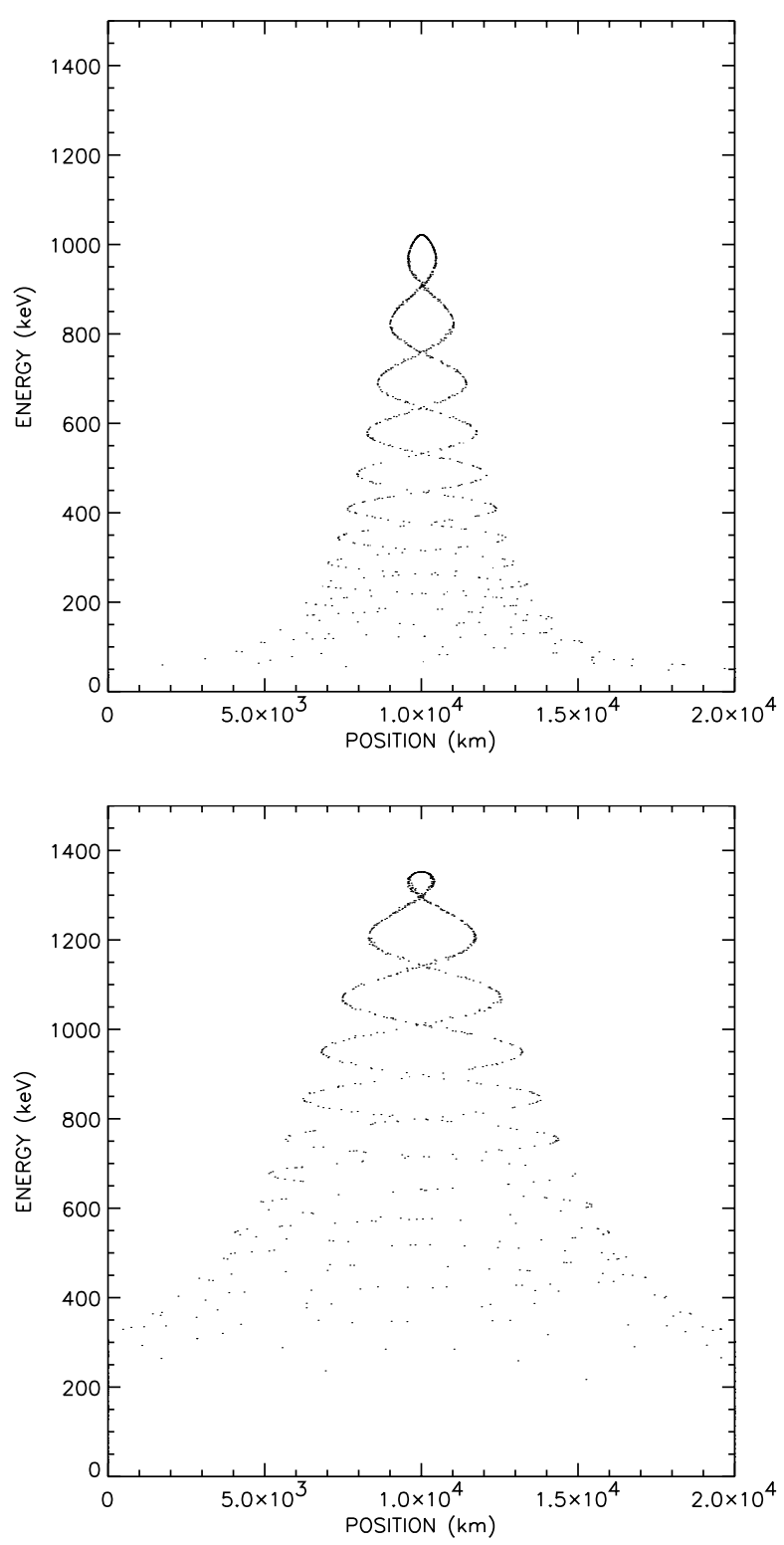

Fig. 5. The distribution of numerical electrons in energy-position space. Each point in the figure corresponds to an individual numerical electron. Positions are expressed along the magnetic field line in the collapsing trap. Upper panel: the state at $t_{0}^{100}+1 \mathrm{~s}$. Lower panel: the state at $t_{0}^{100}+4 \mathrm{~s}$. The initial energy of the electrons is $28.4 \mathrm{keV}$. The initial trap ratio is $R=100$.

While for the case without collisions the total number of numerical electrons in the trap and that with non-zero energy are the same, for the case with collisions these numbers differ because many electrons are stopped in the trap (they have zero energy in our model).

This figure shows that in both cases (with and without collisions $)$ at the very beginning $(t \approx 0.2-0.4 \mathrm{~s})$ the electrons having low pitch angles in the initial state immediately escape from the trap. But their energy is relatively low, it corresponds to the initial energy (obtained by the primary acceleration process) in the case without collisions or even lower in the case with collisions. Simultaneously, the high-pitch-angle electrons begin to be accelerated. While the electrons with $\mu=0$ obtain the
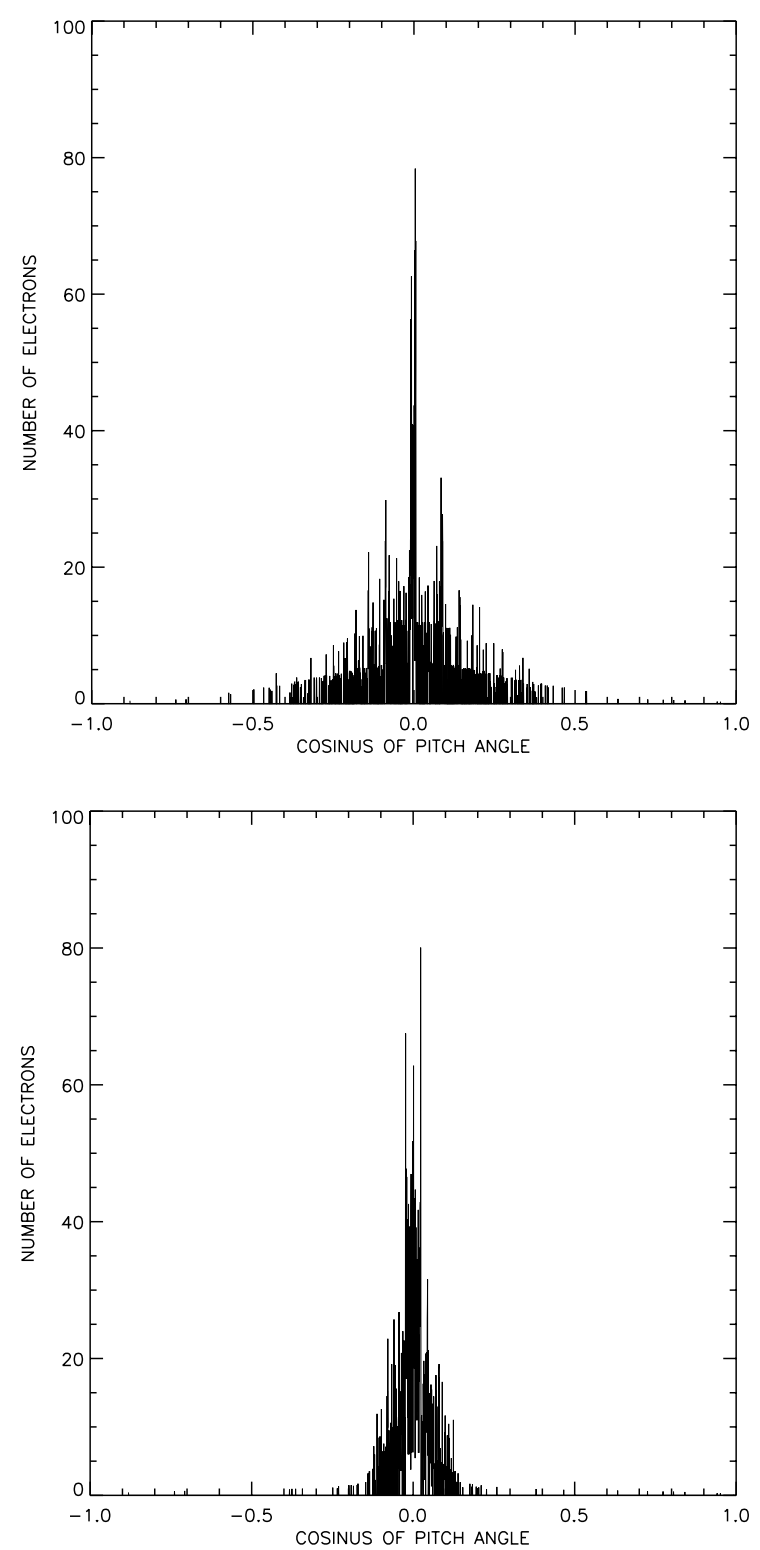

Fig. 6. The distribution of $\mu$ in the collapsing magnetic trap at $t_{0}^{100}+1 \mathrm{~s}$ (upper panel), and at $t_{0}^{100}+4 \mathrm{~s}$ (lower panel). The initial energy is $28.4 \mathrm{keV}$. The initial trap ratio is $R=100$. The electron number includes the weight of the numerical electrons.

maximum energy at the end of the collapse (for times $t \geq 4 \mathrm{~s}$ ), the maximum energy of all trapped electrons is respectively at $t=3 \mathrm{~s}$ and $t=1.9 \mathrm{~s}$ for the case without and with collisions. These maxima lie before the end of the trap collapse because the number of trapped electrons is decreasing and also in the case with collisions the electrons lose energy due to these collisions. But at these times the energy flux out of the trap is still increasing reaching the maximum respectively at $t=5 \mathrm{~s}$ and $t=6 \mathrm{~s}$ for the case without and with collisions. This is due to the trap ratio decreasing $(R \rightarrow 1)$ and more and more energetic electrons with higher pitch angles are able to escape. But their number is decreasing.

After this, we analyzed the effect of Coulomb collisions on the energy spectrum. For this purpose, we injected into the collapsing magnetic trap electrons with energies 5, 6, 7, 8, 9, and 

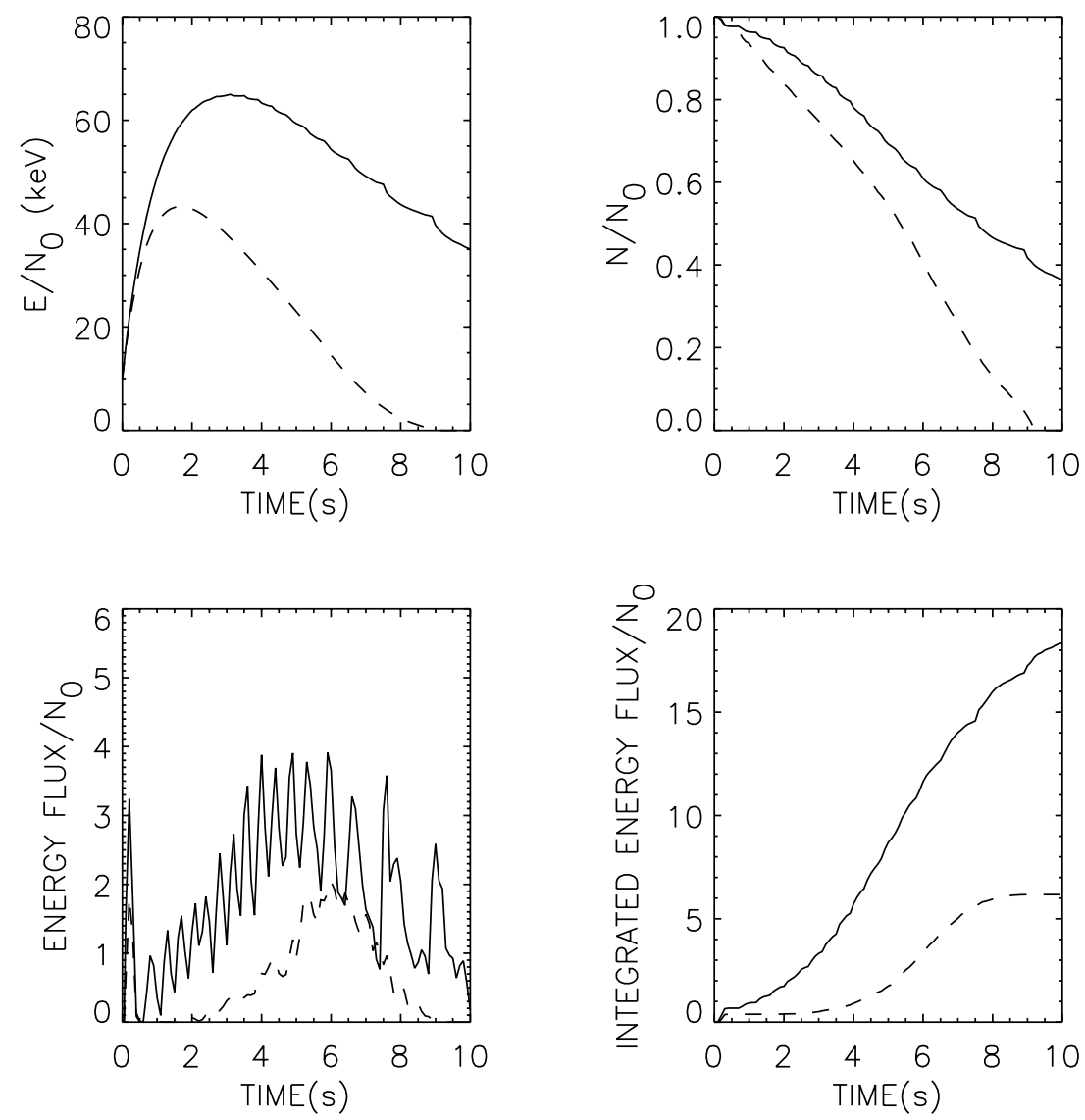

Fig. 7. The time evolution of the energy of electrons in the trap averaged by $N_{0}$ (the number of electrons in the trap in the initial state) in keV, and that of the number of electrons (with non-zero energy) in the trap averaged by $N_{0}$, the energy flux (erg $\mathrm{cm} \mathrm{s}^{-1}$ ) and integrated energy flux (erg cm) averaged by $N_{0}$ at the end point (footpoint) of the trap. The full lines express the case without collisions and dashed lines the case with collisions. The initial trap ratio is $R=10$.

$10 \mathrm{keV}(6 \times 1000$ numerical electrons - see the asterisks in Fig. 8) giving them additional weighting corresponding to the power-law distribution function $F_{\text {init }}(E) \sim\left(E_{0} / E\right)^{3}$. When we use this distribution, the acceleration process in the collapsing magnetic trap (without the collisions) gives the energy spectrum expressed by the full line in Fig. 8. As can be seen in this figure the maximum energy ratio $E_{\text {end }} / E_{0}$ is 10 , which corresponds to the initial trap ratio of $R=10$. This maximum energy gain was obtained by the electrons with $\mu=0$ in the initial state. Other electrons with $\mu \neq 0$ gained less energy as shown by the distribution of electrons at lower energies. Then the Coulomb collisions corresponding to the density model according to relation (9) were included and the resulting spectrum is shown in Fig. 8 by a dashed line. As expected the energies of all electrons are shifted to lower values, especially for low initial energies, e.g. $5 \mathrm{keV}$.

Another possible way to describe the behaviour of electrons in the collapsing trap is to compute their hard X-ray emissivity. This even gives us a possibility to compare these processes with observations.

As we know at any time the positions of numerical electrons and their weights, the hard X-ray emissivity $F_{x}$ at different positions along the trap can be computed as the sum of the contributions of single electrons as follows:

$F_{x}\left(E_{x}\right)=\sum n_{\mathrm{p}} v \sigma_{\mathrm{B}}\left(E_{x}, E\right)$,

where $n_{\mathrm{p}}$ is the number density of ambient protons, $v$ is the electron velocity, $E_{x}$ and $E$ are respectively the radiation and electron energies, and $\sigma_{\mathrm{B}}$ is the bremsstrahlung cross section (Brown 1971; Tangberg-Hanssen \& Emslie 1988):

$\sigma_{\mathrm{B}}\left(E_{x}, E\right)=\frac{7.9 \times 10^{-25}}{E_{x} E} \ln \left[\frac{1+\sqrt{\left(1-\frac{E_{x}}{E}\right)}}{1-\sqrt{\left(1-\frac{E_{x}}{E}\right)}}\right]$,

with $E_{x}$ and $E$ in $\mathrm{keV}$.

Using these relations and output data of the numerical simulation for $R=10$ and the initial electron energy $10 \mathrm{keV}$, the hard X-ray spectra were computed and two of them, at $1.9 \mathrm{~s}$ and $6.0 \mathrm{~s}$ during the magnetic trap collapse, are presented in Fig. 9. The lines for the different spectra correspond to the X-ray spectra from different parts of the collapsing trap: the full line corresponds to the central region of the trap $x=(4-5) \times L / 9$, the dotted line to the region $x=(3-4) \times L / 9$ or $x=(5-6) \times L / 9$, the dashed line to the region $x=(2-3) \times L / 9$ or $x=(6-7) \times L / 9$, the dotted-dashed line to the region $x=(1-2) \times L / 9$ or $x=(7-8) \times L / 9$, and the dot-dot-dot-dashed line to the region 


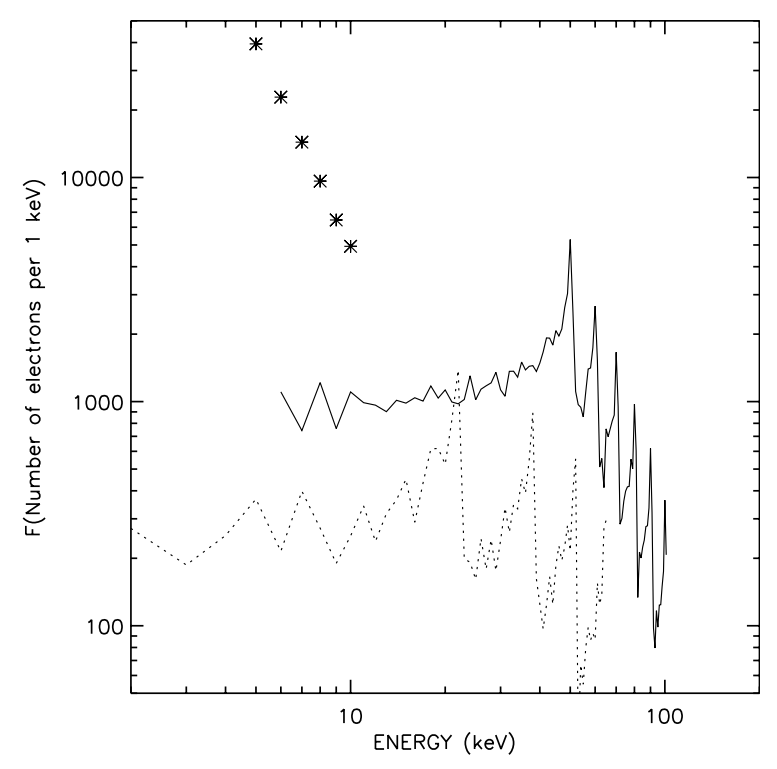

Fig. 8. The distribution function of numerical electrons per $1 \mathrm{keV}$ in the initial state (asterisks) and after $4 \mathrm{~s}$ (full line) for the initial trap ratio $R=10$ in the case without collisions. The dashed line expresses the state after $4 \mathrm{~s}$ but in the case with collisional losses.

$x=(0-1) \times L / 9$ or $x=(8-9) \times L / 9$, where $L$ is the length of the trap, for two times $t=1.9 \mathrm{~s}$ (upper) and $t=6 \mathrm{~s}$ (lower).

As can be seen, the maximum of the X-ray emission in the flare loop is always in the loop centre (full lines in Fig. 9). While at $t=1.9 \mathrm{~s}$ (which corresponds to the time at which the maximum energy of the trapped electrons is highest (see Fig. 7, the case with collisions)), the X-ray intensity as well as the energy extent of the spectrum decrease sharply from the top of the loop to the trap footpoints (upper part of Fig. 9), at $t=6.0 \mathrm{~s}$ (at the time of maximum energy flux outflow from the trap at its end points) the X-ray intensity maximum remains at the top of the loop, but the intensity differences and the energy extent of the spectrum along the loop are much lower. This shows that at this time, when the trap ratio is nearly $R=1$, energetic electrons are nearly homogeneously distributed along the "trap".

Because the distribution of the X-ray emission along the magnetic field line at $t=1.9 \mathrm{~s}$ corresponds to greater heights in the cusp-structure than that at $t=6 \mathrm{~s}$, this result indicates a formation of the X-ray loop-top source above the flare loop. Although the plasma density at the centre of the magnetic field line at $t=1.9 \mathrm{~s}$ is lower than that at $t=6 \mathrm{~s}$, due to the accumulation of a large amount of high-energy electrons in the centre of the magnetic field lines at $t=1.9 \mathrm{~s}$, the intensity here is greater than that at $t=6 \mathrm{~s}$ (compare the spectra (full lines) in the upper and lower panels of Fig. 9). For physical reasons the same result is expected also for the power-law distribution function of injected electrons.

\section{Adiabatic heating}

Not only superthermal particles as studied above, but all particles (electrons, protons, ions) including thermal particles suffer the influence of the betatron electric field $(\nabla \times \boldsymbol{E}=-\partial(\boldsymbol{B}) / \partial t)$. For thermal particles this process is called adiabatic heating
(Emslie 1981). The betatron electric field always increases the component of the momentum perpendicular to the magnetic field or for the thermal particles considered now it increases the perpendicular temperature $T_{\perp}$; the parallel temperature $T_{\|}$ remains unaltered. If the isotropization process due to Coulomb collisions is fast enough (the time for the isotropization is $\tau_{\mathrm{i}} \sim 5 \times 10^{-2} T^{3 / 2} \mathrm{n}^{-1} \mathrm{~s}-$ Spitzer 1962) then thermal particles are described by one temperature $T$ only which changes as

$\frac{1}{T_{0}} \frac{\mathrm{d} T(t)}{\mathrm{d} t}=\frac{2}{3} \frac{1}{B_{0}} \frac{\mathrm{d} B(t)}{\mathrm{d} t}+H-Q$,

where $H$ is the additional heating, and $Q$ all types of losses (e.g. due to the expansion of a hot region along magnetic field lines, due to thermal conduction or radiative losses). Otherwise, two different temperatures $T_{\perp}$ and $T_{\|}$need to be considered.

Because the maximum change of the magnetic field is in the centre of the collapsing magnetic trap that is where the maximum increase of the temperature can be expected. But, as can be seen from relation (12), the resulting temperature depends on the additional heating and losses.

\section{Radio diagnostics of the magnetic trap collapse}

Except for dense plasmas with fast isotropization the betatron process yields plasmas or their superthermal parts with $T_{\perp} \gg T_{\|}$. Just such plasmas are unstable to upper-hybrid waves at positions where the so called double resonance condition is fulfilled (Ledenev et al. 2001):

$\omega_{\mathrm{UH}}=\sqrt{\omega_{\mathrm{pe}}^{2}+\omega_{\mathrm{Be}}^{2}} \approx s \omega_{\mathrm{Be}}$,

where $\omega_{\mathrm{UH}}, \omega_{\mathrm{pe}}$, and $\omega_{\mathrm{Be}}$ are the upper-hybrid, electron plasma and electron gyro frequencies, respectively, $s$ is the harmonic number.

In our model, where the magnetic field and density are described according to the relations (3) and (9), the positions where the resonance condition (at different times during the collapse of one specific magnetic field line) is fulfilled can be calculated as

$\cos \frac{2 \pi x}{L}=1+\frac{\frac{A}{s^{2}-1}-1}{\frac{1}{2} \exp \left(-t / t_{\mathrm{c}}\right)}$

where

$A=\frac{4 \pi n_{0} m_{\mathrm{e}} c^{2}}{B_{0}^{2}}$.

For the selected parameters $\left(B_{0}=100 \mathrm{G}, n_{0}=1.4 \times 10^{10} \mathrm{~cm}^{-3}\right.$, $\left.t_{\mathrm{c}}=1 \mathrm{~s}\right)$ the resulting cosine, which expresses the position along the collapsing magnetic line $(\cos 2 \pi x / L=-1$ corresponds to the trap centre $L / 2$ ), vs. time is shown for three harmonic numbers $(s=4,5,6)$ in Fig. 10. Varying the selected parameters $\left(B_{0}\right.$ and $\left.n_{0}\right)$ we can obtain not only the resonance lines for different times $t$ and positions $(\cos (2 \pi x / L))$, but we also can obtain more harmonic lines when we decrease, for example, the magnetic field $B_{0}$. However, clearly the lower magnetic field $B_{0}$ in the trap means a less efficient betatron process, while the initial magnetic field profile remains the same. An interesting aspect of the selected model is that the resonance line 

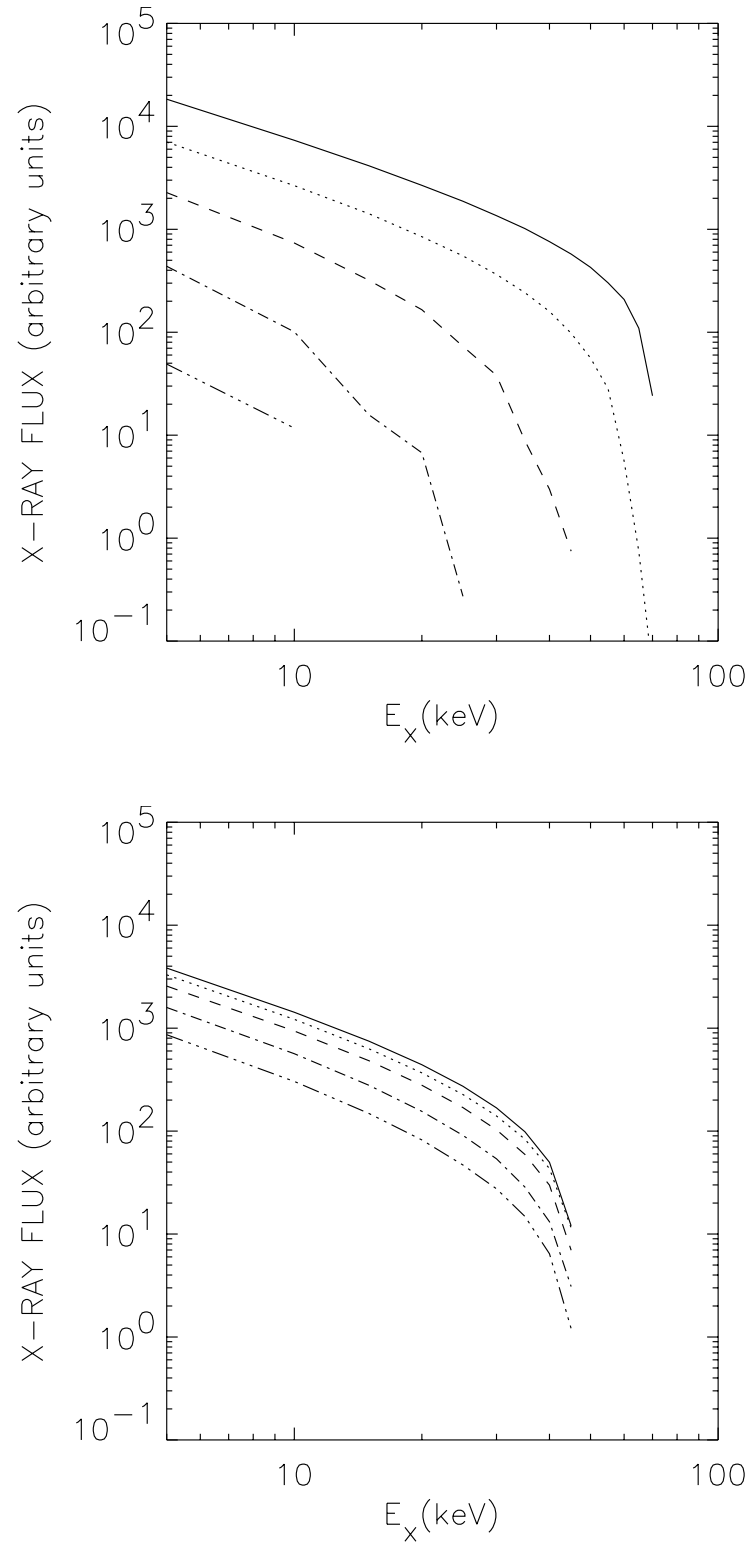

Fig. 9. The X-ray spectra from five different parts along the collapsing trap: the full line corresponds to the central region of the trap $x=$ $(4-5) \times L / 9$, the dotted line to the region $x=(3-4) \times L / 9$ or $x=$ $(5-6) \times L / 9$, the dashed line to the region $x=(2-3) \times L / 9$ or $x=$ $(6-7) \times L / 9$, the dotted-dashed line to the region $x=(1-2) \times L / 9$ or $x=(7-8) \times L / 9$, and the dot-dot-dot-dashed line to the region $x=$ $(0-1) \times L / 9$ or $x=(8-9) \times L / 9$, where $L$ is the length of the trap, for two times: $t=1.9 \mathrm{~s}$ (upper) and $t=6 \mathrm{~s}$ (lower). The initial energy is $10 \mathrm{keV}$, collisions included. The initial trap ratio is $R=10$. The electron number includes the weight of the numerical electrons.

for one specific harmonic number $s$ corresponds to one upperhybrid frequency (in our case $1075 \mathrm{MHz}$ for $s=4,840 \mathrm{MHz}$ for $s=5$, and $691 \mathrm{MHz}$ for $s=6$ ).

From previous analysis we know that the betatron process is the most efficient in the centre of the collapsing trap and also that here the difference between $T_{\perp}$ and $T_{\|}$has its maximum. If moreover it happens that the resonance lines are here then the upper-hybrid waves are generated and after their transformation into electromagnetic waves the dm-continua, zebras or

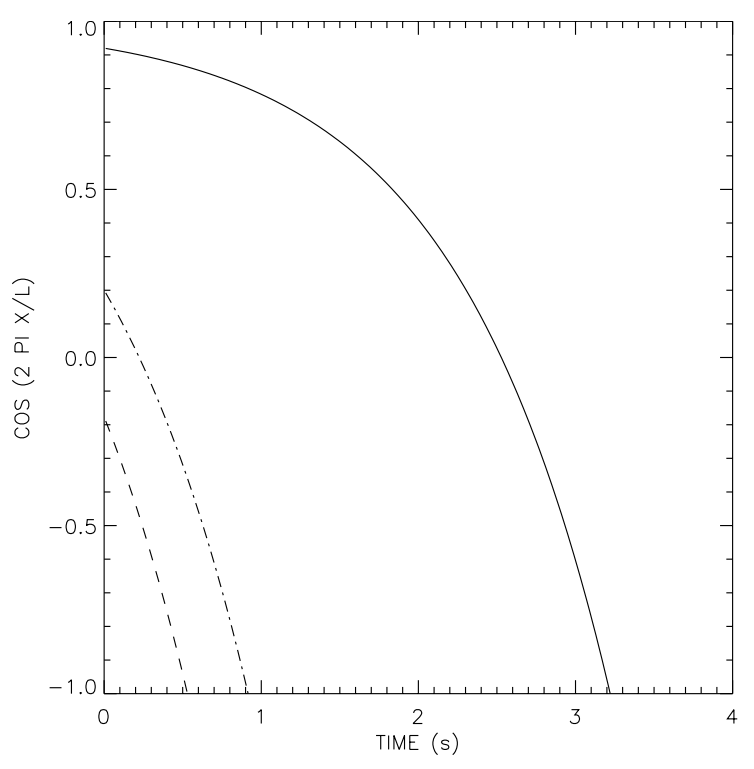

Fig. 10. The plasma resonance lines expressed in $\cos (2 \pi x / L)-$ time space. The full line corresponds to the harmonic number $s=4$, the dashed-dotted line to $s=5$, and the dashed line to $s=6 . B_{0}=100 \mathrm{G}$ and $n_{0}=1.4 \times 10^{10} \mathrm{~cm}^{-3}$ were used.

individual narrowband lines (Chernov et al. 1998), generated in the collapsing magnetic trap, can be observed.

\section{Discussion and conclusions}

The model, which schematically describes the evolution of the magnetic field line in the cusp-structure of a flare, was used here to study an associated acceleration process. Let us first summarize the physical aspects of the simulations:

1. In this secondary acceleration process electrons are accelerated if their energy gains are greater than collisional losses. Because the electron collisional losses are inversely proportional to its energy this means that for a specific plasma density the acceleration of electrons is possible only for energies above some threshold. For example, in our case of a collapsing magnetic trap (during about $4 \mathrm{~s}$ ) for a compressed plasma with a characteristic density of $10^{10} \mathrm{~cm}^{-3}$ the acceleration is possible if the energy of injected electrons (from the primary acceleration process) is above $\sim 5 \mathrm{keV}$.

2. The acceleration is the most efficient at the positions of the largest magnetic field changes; i.e. in the centre of the collapsing magnetic trap, for the electrons having only a perpendicular momentum $\left(p_{\|}=0\right)$ in the initial state. The acceleration of the electrons with a non-zero parallel momentum component is less efficient because these electrons move to the regions with lower magnetic field changes. With an increase of the absolute value of the cosine of the electron pitch angle $|\mu|$ in the initial state the distance of the mirror reflection point for electrons increases and their energy gain decreases.

3. Betatron acceleration in the collapsing trap essentially decreases $\mu$, especially in the central part of the trap, thus accumulating the most energetic electrons here. 
4. For a $\delta$ distribution function (in energy) of injected electrons it was found that the maximum of the X-ray emission is at the top of the collapsing magnetic line. Furthermore it was found that the X-ray emission intensity here is greater for shorter times during the magnetic field line evolution, i.e. at higher heights in the cusp structure of the flare. This indicates the formation of an X-ray source above the flare loop. For physical reasons the same result is expected for the power-law distribution function of injected electrons. A full computation with a power-law distribution function, which is more realistic, but which needs a much greater amount of numerical electrons, can be done in a future model describing the evolution of the magnetic field line in a more precise way.

5. If the electrons injected into the collapsing trap (we expect these electrons from the above located reconnection process) have an isotropic distribution then the electrons with $\mu$ close to 1 and -1 immediately escape from the trap. These electrons correspond to those analyzed in the time-of-flight modelling made by Aschwanden et al. (1996). Clearly, their energy, obtained by the primary acceleration process, may be higher than considered in the present simulations. On the other hand, the electrons with higher energies (after acceleration by the betatron acceleration process) escape later during the trap collapse when the trap ratio decreases to $R \rightarrow 1$. This is the reason that first there appears a maximum of the total electron energy in the trap followed by the maximum of the energy flux out of the trap (second maximum in Fig. 7).

6. All these results are valid also for the proton acceleration, except that the proton scattering for fast protons can be neglected (see Emslie 1978).

Now the question arises what the relevance is of the simulated processes to those observed. First, let us mention limitations for a direct comparison of the model results with observations:

a) The magnetic field and plasma density along the magnetic field line in the collapsing trap in the cusp-structure are expressed only approximately. However, it can be expected that similar results can be obtained also for a class of symmetric functions (around $x=L / 2$ ) replacing the cosine function in relation (3). For future and more realistic computations the magnetic field variations need to be computed by 2-D or 3-D MHD simulations of these processes.

b) In the present paper we studied acceleration processes only along one magnetic field line in the collapsing trap. In reality, in a steady state the flare cusp-structure is formed by a sequence of such moving magnetic field lines.

c) The present model is a simple one. Further processes can be included. For example, the reconnection outflow plasma with a speed close to the Alfven speed may be unstable, an MHD turbulence may be generated and electrons are then effectively accelerated in this MHD turbulence (LaRosa et al. 1996). Furthermore, the outflowing plasma can generate a termination shock at the top of growing flare loops which can also accelerate electrons and thus make the reality more complex than assumed in the present model. d) The approach used here is 2-dimensional. In the 3-D case, due to an additional magnetic field component, the trap ratio $R$ and its change during the "post-flare" loop formation is probably lower and thus the betatron acceleration less efficient. Nevertheless this betatron process, which depends on real flare conditions, can play an important role also in the 3-D case.

e) The density in the flare loop (the final state of the moving magnetic field line) was assumed to be nearly constant. Density variations caused by e.g. an evaporation process, which change collisional processes along the flare loop and also change the resulting spatial distribution of the X-ray emission, were not considered.

All these effects limit direct comparison with observations. Nevertheless, the presented results lead to the following conclusions:

This type of acceleration is a natural aspect of the motion of the plasma and magnetic field lines in the cusp-structure topology.

This secondary acceleration process can be very efficient and due to accumulation of high-energy electrons in the centre of the collapsing trap it give us a new possibility to explain the formation of a loop-top hard X-ray source. Among several models of this loop-top source considering a super hot plasma at the termination shock (Tsuneta et al. 1997), MHD turbulence (Miller et al. 1996; Petrosian et al. 2002), there is one model (Fletcher \& Martens 1998) which is very similar to the present one. While in the model by Fletcher \& Martens (1998) the high-pitch-angle electrons are injected into the trap by the primary acceleration process, in the present model these electrons (with high pitch angles) result from the betatron acceleration process. In reality both these processes can work together.

While the acceleration works for a sufficiently fast collapse and sufficiently high energies of the injected electrons, adiabatic heating works without such limitations. The collapsing trap can easily heat the plasma to high temperatures; but with limitations given by cooling processes (see relation 12). This process can form a region with super hot plasma at the loop-top.

An important criterion of the role of the magnetic trap collapse in real flares is the timing of X-ray and radio bursts. It is known that some short-lasting X-ray and radio bursts are associated to within fractions of the second (see the time-of-flight analysis made by Aschwanden et al. 1996), others show a delay of the radio bursts relative to the X-ray bursts of up to $14 \mathrm{~s}$ (see e.g. Fárnik et al. 2001 and references therein). Considering these observations from the point of view of the described magnetic trap collapse, the simultaneously observed X-ray and radio bursts (within fractions of the second) are generated by electrons accelerated by the primary acceleration process (in the reconnection place), propagating along the magnetic field lines of the trap, and immediately escaping the trap. These electrons are not accelerated by the magnetic field collapse. In our simulations this group of electrons is mono-energetic with an energy corresponding to the initial electron energy. If this group of electrons has some energy distribution then the electrons with higher energy reach the dense chromospheric layers 
(where the thick-target hard X-ray emission is generated) earlier than the electrons with smaller energy, in agreement with the time-of-flight measurements made by Aschwanden et al. (1996).

Furthermore, the processes under study can explain some delayed radio bursts in the decimetric frequency range (generated by the trapped high-energy electrons), and also some delayed component of the hard X-ray emission (generated by the high-energy electrons escaping from the collapsing trap and penetrating dense chromospheric layers).

On the other hand, a comparison of timing in specific events can be used for a modification of the functional expressions in the model relation (3).

In the paper by Nakajima \& Yokoyama (2002) the observation of the non-thermal ejection at $17 \mathrm{GHz}$ by the Nobeyama radioheliograph was described and its model suggested. Here we propose the processes under study as a further possibility of how to explain the non-thermal collimated ejection. Namely, if we assume a reconnection site moving fast enough upwards into the corona then the underlying flare loops grow fast in the upwards direction and the collapsing trap accelerates electrons for the gyro-synchrotron emission observed at $17 \mathrm{GHz}$.

We have shown that a magnetic trap collapse accelerates electrons with a distribution function appropriate for radio emission at locations where the so-called double resonance condition is fulfilled. Therefore, we expect that the magnetic field collapse can be associated with observations of radio continua, narrowband lines or zebras in the decimetric frequency range.

Acknowledgements. M.K. would like to thank the Institute of Space and Astronautical Science at Sagamihara, Japan for inviting him as a guest professor (July-September 2003) and providing him with financial support. He is grateful to his colleagues for friendly and generous hospitality during his stay in Japan. This research was partly supported also by Grant IAA3003202 of the Academy of Sciences of the Czech Republic. The authors thank P. Martens for helpful comments and suggestions.

\section{References}

Aschwanden, M. J. 2002, Space Sci. Rev., 101(1-2)

Aschwanden, M. J., Wills, M. J., Hudson, H. S., Kosugi, T., \& Schwartz, R. A. 1996, ApJ, 486, 398
Bai, T. 1982, ApJ, 259, 341

Berger, J. M., Newcomb, W. A., Dawson, J. M., et al. 1958, Phys. Fluids, 1, 301

Brown, J. C. 1971, Sol. Phys., 18, 489

Brown, J. C., \& Hoyng, P. 1975, ApJ, 200, 734

Bruggmann, G., Vilmer, N., Klein, K.-L., \& Kane, S. R. 1994, Sol. Phys., 149, 171

Cargill, P. J., Goodrich, C. C., \& Vlahos, L. 1988, A\&A, 189, 254

Chernov, G. P., Markeev, A. K., Poquerusse, M., et al. 1998, A\&A, 334,314

Emslie, A. G. 1978, ApJ, 224, 241

Emslie, A. G. 1981, ApJ, 244, 653

Fárnik, F., Garcia, H., \& Karlický, M. 2001, Sol. Phys., 201, 357

Fletcher, L., Martens, P. C. H. 1998, ApJ, 505, 418

Holman, G. D., \& Pesses, M. E. 1983, ApJ, 267, 837

Jakimiec, J. 2002, Proc. 10th European Solar Physics Meeting, Solar Variability: From Core to Outer Frontiers, Prague, Czech Republic, 645

Karlický, M. 1993, Sol. Phys., 145, 137

Karlický, M., \& Henoux, J. C. 1993, A\&A, 278, 627

LaRosa, T. N., \& Moore, R. L. 1993, ApJ, 418, 912

LaRosa, T. N., Moore, R. L., Miller, J. A., \& Shore, S. N. 1996, ApJ, 467,454

Ledenev, V. G., Karlický, M., \& Fu, Q. 2001, Sol. Phys., 202, 71

Litvinenko, Y. E. 1996, ApJ, 462, 997

Masuda, S., Kosugi, T., Tsuneta, S., \& Hara, H. 1996, Adv. Space Res., 17, 63

Miller, J. A., LaRosa, T. N., \& Moore, R. L. 1996, ApJ, 461, 445

Nakajima, H., \& Yokoyama, T. 2002, ApJ, 570, L41

Petrosian, V., Donaghy, T. Q., \& McTierman, J. M. 2002, ApJ, 569, 459

Priest, E. R. 1982, Solar Magnetohydrodynamics (D. Reidel Publ. Comp.), 104

Priest, E. R., \& Forbes, T. 2000, Magnetic Reconnection: MHD Theory and Applications (Cambridge, UK: Cambridge University Press)

Somov, B. V., \& Kosugi, T. 1997, ApJ, 485, 859

Spitzer, L. W. 1962, Physics of Fully Ionized Gases (New York: Interscience)

Swann, W. F. G. 1933, Phys. Rev., 43, 217

Tangberg-Hanssen, E., \& Emslie, A. G. 1988, The Physics of Solar Flares, Cambridge Astrophys. Ser. (Cambridge: Cambridge Univ. Press)

Tsuneta, S., \& Naito, T. 1998, ApJ, 495, L67

Tsuneta, S., Masuda, S., Kosugi, T., \& Sato, J. 1997, ApJ, 478, 787 